VOL. 55 (1997) [239-248]

\title{
A KOROVKIN TYPE THEOREM FOR WEIGHTED SPACES OF CONTINUOUS FUNCTIONS
}

\author{
WALTER ROTH
}

\begin{abstract}
We prove a Korovkin type approximation theorem for positive linear operators on weighted spaces of continuous real-valued functions on a compact Hausdorff space $X$. These spaces comprise a variety of subspaces of $C(X)$ with suitable locally convex topologies and were introduced by Nachbin 1967 and Prolla 1977. Some early Korovkin type results on the weighted approximation of real-valued functions in one and several variables with a single weight function are due to Gadzhiev 1976 and 1980.
\end{abstract}

\section{Weighted Spaces of Functions}

Throughout this paper, let $X$ be a locally compact Hausdorff space. A real-valued function $f$ on $X$ is said to vanish at infinity if for every $\varepsilon>0$ the set $\{x \in X|| f(x) \mid$ $\geqslant \varepsilon\}$ is relatively compact. As usual, we denote by

$C(X)$ the space of all continuous real-valued functions on $X$,

$C_{B}(X)$ the space of all bounded functions in $C(X)$,

$C_{0}(X)$ the space of all functions in $C(X)$ that vanish at infinity,

$C_{c}(X)$ the space of all functions in $C(X)$ with compact support.

$A$ variety of norms and seminorms may be considered on these spaces. The supremum norm is generally available for $C_{B}(X), C_{0}(X)$ and $C_{c}(X)$. Korovkin type approximation theorems have been developed for $C_{0}(X)$ endowed with the supremum norm, by Bauer and Donner [2] and other authors. In the following we shall present a general approach that deals simultaneously with a variety of subspaces of $C(X)$, carrying different locally convex topologies. We shall use the concept of weighted spaces of functions as developed by Nachbin. We give a short introduction to the general theory. For details and proofs we globally refer to Nachbin's [5] fundamental work and the monograph by Prolla [6].

A family $\mathcal{W}$ of non-negative upper semicontinuous functions on $X$ is called a family of weights if for all $w_{1}, w_{2} \in \mathcal{W}$ there are $w_{3} \in \mathcal{W}$ and $\rho>0$ such that

$$
w_{1} \leqslant \rho w_{3} \text { and } w_{2} \leqslant \rho w_{3}
$$

Received 4th April, 1996

Copyright Clearance Centre, Inc. Serial-fee code: 0004-9729/97 \$A2.00+0.00. 
With any family of weights $\mathcal{W}$ we associate the subspace of $C(X)$

$$
C_{\mathcal{W}}(X)=\{f \in C(X) \mid w f \text { vanishes at infinity for all } w \in \mathcal{W}\}
$$

Together with the locally convex topology generated by the seminorms

$$
p_{w}(f)=\sup \{|w f(x)| \mid x \in X\}
$$

for $w \in \mathcal{W}$ and $f \in C_{\mathcal{W}}(X)$ we call $C_{\mathcal{W}}(X)$ a weighted space of functions. If for every $x \in X$ there is some $w \in W$ such that $w(x) \neq 0$, then the topology of $C_{\mathcal{W}}(X)$ is obviously Hausdorff. We shall mention some examples for weighted spaces of functions.

EXAMPLE 1.1.

(a) If $\mathcal{W}$ consists of the constant function $w \equiv 1$, then $C_{\mathcal{W}}(X)=C_{0}(X)$, and $p_{w}$ is the supremeum norm.

(b) If $\mathcal{W}=C_{0}^{+}(X)=\left\{w \in C_{0}(X) \mid w \geqslant 0\right\}$, then $C_{\mathcal{W}}(X)=C_{B}(X)$, but the weighted topology (called the strict topology) is generally coarser than the supremum norm topology on $C_{B}(X)$.

(c) If $\mathcal{W}$ consists of the characteristic functions of all compact subsets of $X$, then $C_{\mathcal{W}}(X)=C(X)$ with the topology of compact convergence.

(d) If $\mathcal{W}$ consists of the characteristic functions of all finite subsets of $X$, then $C_{W}(X)=C(X)$ with the topology of pointwise convergence.

(e) If $\mathcal{W}=C^{+}(X)=\{w \in C(X) \mid w \geqslant 0\}$, then $C_{\mathcal{W}}(X)=C_{c}(X)$. The weighted topology is generally finer than the supremum norm but coarser than the inductive limit topology on $C_{c}(X)$.

Example 1.1(d) shows in particular that weighted spaces of functions need not be complete. Note that $C_{c}(X)$ is a dense subspace of $C_{\mathcal{W}}(X)$ for any choice of the family of weights $\mathcal{W}$.

The following characterisation of the dual of a weighted space of functions may be found in Prolla's monogragh [6]. By $M_{B}(X)$ we denote the space of all finite regular Borel measures on $X$, and by $M_{B}^{+}(X)$ the positive cone in $M_{B}(X)$. The norm of $\mu \in M_{B}(X)$ is defined as $\|\mu\|=|\mu|(X)$. By the Riesz Representation Theorem every bounded linear functional on $C_{c}(X)$ endowed with the supremum norm, may be uniquely represented as a measure $\mu \in M_{B}(X)$. For weighted spaces in general, every continuous linear functional on $C_{\mathcal{W}}(X)$ is seen to be continuous with respect to the inductive topology of the subspace $C_{c}(X)$, hence may be represented for the functions in $C_{\mathcal{W}}(X)$ by a (not necessarily bounded) Borel measure on $X$.

For all $f \in C_{\mathcal{W}}(X)$ and $w \in \mathcal{W}$, the function $w f$ is integrable with respect to every $\mu \in M_{B}(X)$. Thus by $w \mu$ we denote the linear functional

$$
f \mapsto \mu(w f): C_{w}(X) \rightarrow \mathbb{R} .
$$


As $|w \mu(f)| \leqslant\|\mu\| p_{w}(f)$, the functional $w \mu$ is in the dual of $C_{\mathcal{W}}(X)$. Moreover, it may be seen that the functionals of this type constitute the whole dual of $C_{\mathcal{W}}(X)$. For the proof of this fact we refer to Prolla [6, Theorem 5.42]:

Theorem 1.2. The dual of $C_{\mathcal{W}}(X)$ may be identified with the space of all functionals $w \mu$, where $w \in \mathcal{W}$ and $\mu \in M_{B}(X)$; more precisely: For $w \in \mathcal{W}$ the polar of the 0 -neighbourhood $V=\left\{f \in C_{W}(X) \mid p_{w}(f) \leqslant 1\right\}$ is given by

$$
V^{\circ}=\left\{w \mu \mid \mu \in M_{B}(X),\|\mu\| \leqslant 1\right\} .
$$

Positive linear functionals on $C_{\mathcal{W}}(X)$ are represented by positive measures $\mu \in$ $M_{B}^{+}(X)$.

Reviewing some of our Examples 1.1, we realise that in (a) the dual of $C_{\mathcal{W}}(X)$ consists of $M_{B}(X)$, in (c) of those measures in $M_{B}(X)$ having compact support, and in (d) of the linear combinations of point evaluations (called simple measures).

\section{The Main Approximation Theorem}

The weighted spaces $C_{\mathcal{W}}(X)$ are endowed with the pointwise order for functions, and a linear operator $T: C_{\mathcal{W}}(X) \rightarrow C_{\mathcal{W}}(X)$ is said to be positive if $T(f) \geqslant 0$, whenever $f \geqslant 0$. A family $\mathcal{T}$ of linear operators on $C_{\mathcal{W}}(X)$ is equicontinuous if for every $w \in \mathcal{W}$ there are $w^{\prime} \in \mathcal{W}$ and $\rho>0$ such that for the corresponding seminorms

$$
p_{w}(T(f)) \leqslant \rho p_{w^{\prime}}(f)
$$

holds for all $f \in C_{\mathcal{W}}(X)$ and $T \in \mathcal{T}$.

Korovkin type theorems deal with approximation processes modelled by equicontinuous nets $\left(T_{\alpha}\right)_{\alpha \in \mathcal{A}}$ of positive linear operators. We write $T_{\alpha}(f) \rightarrow f$ if the net $\left(T_{\alpha}(f)\right)_{\alpha \in \mathcal{A}}$ converges to $f$ in the topology of $C_{\mathcal{W}}(X)$.

For a subset $\mathcal{M}$ of $C_{\mathcal{W}}(X)$, the Korovkin closure $\mathcal{K}(\mathcal{M})$ of $\mathcal{M}$ consists of all functions $f \in C_{\mathcal{W}}(X)$ such that $T_{\alpha}(f) \rightarrow f$ whenever $\left(T_{\alpha}\right)_{\alpha \in \mathcal{A}}$ is an equicontinuous net of positive linear operators on $C_{\mathcal{W}}(X)$ and $T_{\alpha}(g) \rightarrow g$ holds for all $g \in \mathcal{M}$. A Korovkin system for $C_{\mathcal{W}}(X)$ is a subset $\mathcal{M} \subset C_{\mathcal{W}}(X)$ such that $\mathcal{K}(\mathcal{M})=C_{\mathcal{W}}(X)$. By $\operatorname{span}(\mathcal{M})$ we denote the linear span of $\mathcal{M}$; that is, the subspace of $C_{\mathcal{W}}(X)$ generated by $\mathcal{M}$.

Now we are ready to formulate our main result. For the special case of $C_{0}(X)$ with the supremum norm, that is, the case of our Example 1.1(a), it is due to Bauer and Donner [2].

TheOREM 2.1. Let $X$ be a locally compact Hausdorff space, and let $\mathcal{W}$ be a family of weight functions on $X$. Let $\mathcal{M}$ be a subset of $C_{W}(X)$. For a function $f \in C_{\mathcal{W}}(X)$ the following are equivalent:

(a) $f \in \mathcal{K}(\mathcal{M})$. 
(b) For every $x \in X$ such that $w(x)>0$ for at least one weight function $\boldsymbol{w} \in \mathcal{W}$,

$$
\begin{aligned}
f(x) & =\sup _{\substack{w \in \mathcal{W} \\
\varepsilon>0}} \inf \{g(x) \mid g \in \operatorname{span}(\mathcal{M}), \quad w f \leqslant w g+\varepsilon\} \\
& =\inf _{\substack{w \in \mathcal{W} \\
\varepsilon>0}} \sup \{g(x) \mid g \in \operatorname{span}(\mathcal{M}), \quad w g \leqslant w f+\varepsilon\} .
\end{aligned}
$$

(c) For every $x \in X$ such that $w(x)>0$ for at least one weight function $w \in \mathcal{W}$, and for every $\mu \in M_{B}^{+}(X)$ and $w \in \mathcal{W}$

$$
\mu(w g)=g(x) \text { for all } g \in \mathcal{M} \text { implies } \mu(w f)=\xi(x) \text {. }
$$

Proof: $(\mathrm{a}) \Rightarrow(\mathrm{c})$ : Let us assume that (c) fails for the function $f \in C_{W}(X)$, the point $x \in X$, the measure $\mu \in M_{B}^{+}(X)$ and the weight function $w \in \mathcal{W}$; that is, we have

$$
\mu(w g)=g(x) \text { for all } g \in \mathcal{M}, \text { but } \mu(w f) \neq f(x) .
$$

We shall show that (a) fails as well: Let $U_{0}$ be a fixed compact neighbourhood for $\boldsymbol{x}$ and let $\mathcal{U}$ be a basis of open neighbourhoods of $x$ that are all subsets of $U_{0}$. By Urysohn's Lemma, for all $U \in \mathcal{U}$ there are functions $\phi_{U} \in C_{c}(X)$ such that

$$
0 \leqslant \phi_{U} \leqslant 1, \quad \phi_{U}(x)=1, \quad \text { and } \quad \phi_{U} \equiv 0 \text { on } X \backslash U
$$

Using those functions we define operators $T_{U}$ on $C_{W}(X)$ by

$$
T_{U}(h)=h\left(1-\phi_{U}\right)+\mu(w h) \phi_{U}
$$

for $h \in C_{\mathcal{W}}(X)$. Clearly $T_{U}(h) \in C_{\mathcal{W}}(X)$, and the operators $T_{U}$ are linear and positive. Equicontinuity is easily checked: Given $w^{\prime} \in \mathcal{W}$, choose $w^{\prime \prime} \in \mathcal{W}$ and $\rho>0$ such that both

$$
w \leqslant \rho w^{\prime \prime} \text { and } w^{\prime} \leqslant \rho w^{\prime \prime} .
$$

With an upper bound $\sigma>0$ for the upper semicontinuous function $w^{\prime}$ on $U_{0}$, we compute for all $y \in X$ and $U \in \mathcal{U}$

$$
\begin{aligned}
\left|T_{U}(h)(y) w^{\prime}(y)\right| & \leqslant\left(|h(y)|\left(1-\phi_{U}(y)\right)+|\mu(w h)| \phi_{U}(y)\right) w^{\prime}(y) \\
& \leqslant|h(y)| w^{\prime}(y)+\sigma|\mu(w h)| \\
& \leqslant p_{w^{\prime}}(h)+\sigma\|\mu\| p_{w}(h) \\
& \leqslant \rho p_{w^{\prime \prime}}(h)+\sigma \rho\|\mu\| p_{w^{\prime \prime}}(h) \\
& =\rho(1+\sigma\|\mu\|) p_{w^{\prime \prime}}(h) .
\end{aligned}
$$


Thus $p_{w^{\prime}}\left(T_{U}(h)\right) \leqslant \rho(1+\sigma\|\mu\|) p_{w^{\prime \prime}}(h)$. Ordered by reverse inclusion (that is, $U \leqslant V$ if $U \supset V)$, the neighbourhood system $\mathcal{U}$ serves as the index set of the net $\left(T_{U}\right)_{U \in \mathcal{U}}$. For every function $g \in \mathcal{M}$ we observe that

$$
T_{U}(g)(y)=g(y) \text { for all } y \in X \backslash U .
$$

For $y \in U \subset U_{0}$ on the other hand, and for $w^{\prime} \in \mathcal{W}$ and $\sigma>0$ an upper bound for $w^{\prime}$ on $U_{0}$, we verify

$$
\begin{aligned}
\left|T_{U}(g)(y)-g(y)\right| w^{\prime}(y) & =\left|\left(g(y)\left(1-\phi_{U}(y)\right)+\mu(w g) \phi_{U}(y)\right)-g(y)\right| w^{\prime}(y) \\
& \leqslant \phi_{U}(y)|g(x)-g(y)| w^{\prime}(y) \\
& \leqslant \sigma|g(x)-g(y)| .
\end{aligned}
$$

Thus $p_{w^{\prime}}\left(T_{U}(g)-g\right) \leqslant \varepsilon$ for all $U \in \mathcal{U}$ such that $|g(x)-g(y)| \leqslant \varepsilon / \sigma$ for all $y \in U$. This shows that $T_{U}(g) \rightarrow g$ for all $g \in \mathcal{M}$. But for the given function $f$ and the point $x \in X$ we have $T_{U}(f)(x)=\mu(w f) \neq f(x)$. By assumption (c) there exists at least one weight function $w^{\prime} \in \mathcal{W}$ that does not vanish at $x$. This shows $p_{w^{\prime}}\left(T_{U}(f)-f\right) \not 0$, hence $T_{U}(f) \nrightarrow f$, contradicting (a).

(c) $\Rightarrow$ (b): Suppose that the function $f \in C_{\mathcal{W}}(X)$ satisfies (c), and let $x \in X$ be such that $w(x)>0$ for some $w \in \mathcal{W}$. We shall prove that the first equality in (b) holds. The second one may be verified in an analogous way. The inequality

$$
\sup _{\substack{w \in \mathcal{W} \\ \varepsilon>0}} \inf \{g(x) \mid g \in \operatorname{span}(\mathcal{M}), w f \leqslant w g+\varepsilon\} \geqslant f(x)
$$

holds in any case, as for $w \in \mathcal{W}$ such that $w(x)>0$ and $\varepsilon>0$ we realise that

$$
\inf \{g(x) \mid g \in \operatorname{span}(\mathcal{M}), w f \leqslant w g+\varepsilon\} \geqslant f(x)-\varepsilon / w(x) .
$$

For the converse inequality, it suffices to show that for any $w \in \mathcal{W}$ and $\varepsilon>0$ we have

$$
\inf \{g(x) \mid g \in \operatorname{span}(\mathcal{M}), w f \leqslant w g+\varepsilon\} \leqslant f(x) .
$$

As the infimum on the left hand side of the last inequality increases, if we replace $w \in \mathcal{W}$ by a larger weight function $w^{\prime} \in \mathcal{W}$, and as $\mathcal{W}$ is directed upward, we may assume that $w(x)>0$. Next we define a functional $p$ on $C_{\mathcal{W}}(X)$ by

$$
p(h)=\inf \{g(x)+2 \delta / w(x) \mid \delta \geqslant 0, g \in \operatorname{span}(\mathcal{M}), w h \leqslant w g+\delta\} .
$$

As $h(x) \leqslant p(h) \leqslant(2 / w(x)) p_{w}(h)$, the functional $p$ is real-valued on $C_{W}(X)$. It is easily checked to be sublinear, and for $g \in \operatorname{span}(\mathcal{M})$ we realise that $p(g)=g(x)$. Thus, by the Hahn-Banach theorem there is a linear functional $\Phi$ on $C_{\mathcal{W}}(X)$ satisfying

$$
\Phi(h) \leqslant p(h) \text { for all } h \in C_{W}(X) \text { and } \Phi(f)=p(f)
$$


for the given function $f \in C_{\mathcal{W}}(X)$. As $\Phi(h) \leqslant p(h) \leqslant 0$, whenever $h \leqslant 0$, the functional $\Phi$ is positive and continuous with respect to the topology of $C_{W}(X)$, thus may be represented as $w \mu$, where $\mu \in M_{B}^{+}(X)$. By the above, this means $\mu(w g)=g(x)$ for all $g \in \mathcal{M}$, and therefore by (c)

$$
\begin{aligned}
f(x) & =\mu(w f)=\Phi(f)=p(f) \\
& =\inf \{g(x)+2 \delta / w(x) \mid \delta \geqslant 0, g \in \operatorname{span}(\mathcal{M}), w f \leqslant w g+\delta\} .
\end{aligned}
$$

Thus for any choice of $0<\varepsilon^{\prime} \leqslant \varepsilon / w(x)$ we may find $\delta \geqslant 0$ and $g \in \operatorname{span}(\mathcal{M})$ such that

$$
w f \leqslant w g+\delta \text { and } g(x)+2 \delta / w(x) \leqslant f(x)+\varepsilon^{\prime} \leqslant g(x)+\delta / w(x)+\varepsilon^{\prime},
$$

whence $\delta / w(x) \leqslant \varepsilon^{\prime} \leqslant \varepsilon / w(x)$ and $\delta \leqslant \varepsilon$. Summarising, this shows

$$
w f \leqslant w g+\varepsilon \text { and } g(x) \leqslant f(x)+\varepsilon^{\prime},
$$

hence

$$
\inf \{g(x) \mid g \in \operatorname{span}(\mathcal{M}), w f \leqslant w g+\varepsilon\} \leqslant f(x)
$$

as desired.

(b) $\Rightarrow$ (a): Let us assume that (b) holds for the function $f \in C_{\mathcal{W}}(X)$, but that (a) is false. Then there is an equicontinuous net $\left(T_{\alpha}\right)_{\alpha \in \mathcal{A}}$ of positive linear operators on $C_{\mathcal{W}}(X)$ such that

$$
T_{\alpha}(g) \rightarrow g \quad \text { for all } g \in \mathcal{M}, \quad \text { but } \quad T_{\alpha}(f) \nrightarrow f ;
$$

that is, $p_{w}\left(T_{\alpha}(f)-f\right) \nrightarrow 0$ for some weight function $w \in \mathcal{W}$. There are $\delta>0$, a subnet $\left(T_{\beta}\right)_{\beta \in B}$ of $\left(T_{\alpha}\right)_{\alpha \in \mathcal{A}}$ and points $x_{\beta} \in X$ such that

$$
w\left(x_{\beta}\right)\left|T_{\beta}(f)\left(x_{\beta}\right)-f\left(x_{\beta}\right)\right| \geqslant \delta \quad \text { for all } \beta \in \mathcal{B} .
$$

We may assume (after selecting another subnet) that either

$$
w\left(x_{\beta}\right)\left(T_{\beta}(f)\left(x_{\beta}\right)-f\left(x_{\beta}\right)\right) \geqslant \delta \quad \text { or } \quad w\left(x_{\beta}\right)\left(T_{\beta}(f)\left(x_{\beta}\right)-f\left(x_{\beta}\right)\right) \leqslant-\delta
$$

holds for all $\beta \in \mathcal{B}$. We assume the first case, as we continue with our argument. The second case is similar. Let us recall that the operators $\left(T_{\beta}\right)_{\beta \in \mathcal{B}}$ were supposed to be equicontinuous. For the given weight function $w \in \mathcal{W}$ from above there are $w^{\prime} \in \mathcal{W}$ and $\rho>0$ such that

$$
p_{w}\left(T_{\beta}(h)\right) \leqslant \rho p_{w^{\prime}}(h)
$$


for all $h \in C_{\mathcal{W}}(X)$ and $\beta \in \mathcal{B}$. Next we observe that the net $\left(x_{\beta}\right)_{\beta \in B}$ permits the selection of a further subnet $\left(x_{\gamma}\right)_{\gamma \in \mathcal{C}}$ that converges to some $x_{0} \in X$ or to $x_{0}=\infty$. (The latter refers to the one-point compactification on $X$ ). We continue our argument with this subnet. Following condition (b), for any choice of $\varepsilon>0$ and the weight function $w^{\prime} \in \mathcal{W}$ from above there is $g \in \operatorname{span}(\mathcal{M})$ such that

$$
w^{\prime} f \leqslant w^{\prime} g+\varepsilon / \rho, \quad \text { and } \quad w\left(x_{0}\right) g\left(x_{0}\right) \leqslant w\left(x_{0}\right) f\left(x_{0}\right)+\varepsilon .
$$

(The second condition is void for $x_{0}=\infty$ or for $w\left(x_{0}\right)=0$. But our assumption that (a) fails for the function $f$ implies in particular that $\mathcal{W}$ contains a non-zero weight function and that the equalities in (b) hold for at least one point $x \in X$. Thus we may always find a function $g \in \operatorname{span}(\mathcal{M})$ fulfilling the first condition.) With $h=(f-g)^{+}=\sup \{(f-g), 0\} \in C_{\mathcal{W}}(X)$ we have

$$
0 \leqslant w^{\prime} h \leqslant \varepsilon / \rho \text { and } f \leqslant g+h .
$$

Thus $p_{w^{\prime}}(h) \leqslant \varepsilon / \rho$ and $p_{w}\left(T_{\gamma}(h)\right) \leqslant \varepsilon$ for all $\gamma \in \mathcal{C}$ by the above. This shows

$$
T_{\gamma}(f) \leqslant T_{\gamma}(g)+T_{\gamma}(h), \text { hence } w T_{\gamma}(f) \leqslant w T_{\gamma}(g)+\varepsilon .
$$

But we have $T_{\gamma}(g) \rightarrow g$, as $g \in \operatorname{span}(\mathcal{M})$. There is $\gamma_{0} \in \mathcal{C}$ such that $p_{w}\left(T_{\gamma}(g)-g\right) \leqslant \varepsilon$ for all $\gamma \geqslant \gamma_{0}$. Summarising, we obtain for those $\gamma$

$$
w T_{\gamma}(f) \leqslant w T_{\gamma}(g)+\varepsilon \leqslant w g+2 \varepsilon,
$$

and using the selected the points $x_{\gamma} \in X$,

$$
w\left(x_{\gamma}\right) f\left(x_{\gamma}\right)+\delta \leqslant w\left(x_{\gamma}\right) T_{\gamma}(f)\left(x_{\gamma}\right) \leqslant w\left(x_{\gamma}\right) g\left(x_{\gamma}\right)+2 \varepsilon .
$$

To obtain the desired contradiction, now we have to separate our cases:

If $x_{0}=\infty$, then both

$$
w\left(x_{\gamma}\right) f\left(x_{\gamma}\right) \rightarrow 0 \text { and } w\left(x_{\gamma}\right) g\left(x_{\gamma}\right) \rightarrow 0,
$$

a contradiction to our last inequality, if we choose $\varepsilon<\delta / 2$.

If $x_{0} \in X$, but $w\left(x_{0}\right)=0$, then $w\left(x_{\gamma}\right) \rightarrow 0$, as $w$ is non-negatiave and upper semicontinuous. Again, with $\varepsilon<\delta / 2$ we obtain a contradiction.

Finally, if $x_{0} \in X$, and $w\left(x_{0}\right)>0$, we may use the second condition for the choice of the function $g \in \operatorname{span}(\mathcal{M})$ and the continuity of $f$ and $g$ in $x_{0}$ : There is $\gamma_{1} \geqslant \gamma_{0}$ such that for all $\gamma \geqslant \gamma_{1}$

$$
\left|f\left(x_{\gamma}\right)-f\left(x_{0}\right)\right| \leqslant \varepsilon \text { and }\left|g\left(x_{\gamma}\right)-g\left(x_{0}\right)\right| \leqslant \varepsilon
$$


holds. Furthermore we may assume that $w\left(x_{\gamma}\right) \leqslant \sigma$ for some $\sigma>0$. (The function $w$ is bounded above on a compact neighbourhood of $x_{0}$.) Thus for all such $\gamma \geqslant \gamma_{1}$

$$
\begin{aligned}
w\left(x_{\gamma}\right) g\left(x_{\gamma}\right) & \leqslant w\left(x_{\gamma}\right) g\left(x_{0}\right)+\varepsilon \sigma \\
& \leqslant w\left(x_{\gamma}\right) f\left(x_{0}\right)+\varepsilon+\varepsilon \sigma \\
& \leqslant w\left(x_{\gamma}\right) f\left(x_{\gamma}\right)+\varepsilon(1+2 \sigma) \\
& \leqslant w\left(x_{\gamma}\right) g\left(x_{\gamma}\right)+\varepsilon(3+2 \sigma)-\delta,
\end{aligned}
$$

and $\delta \leqslant \varepsilon(3+2 \sigma)$, a contradiction if we choose $\varepsilon$ sufficiently small, thus completing our proof.

An application of Theorem 2.1 yields a Stone-Weierstrass theorem for weighted spaces. The result is well-known for the special case of $C_{0}(X)$ with the supremum norm (see [1, Chapter 4.4]).

CoRollary 2.2. Let $X$ be a locally compact Hausdorff space, and let $\mathcal{W}$ be a family of weight functions on $X$. If $\mathcal{M} \subset C_{\mathcal{W}}(X)$ is a Korovkin system for $C_{\mathcal{W}}(X)$, then the vector sublattice generated by $\mathcal{M}$ is dense in $C_{\mathcal{W}}(X)$.

Proof: Let $\mathcal{M}$ be a Korovkin system for $C_{\mathcal{W}}(X)$. To prove our claim, it suffices to show that every positive function $f \in C_{\mathcal{W}}(X)$ may be approximated in the topology of $C_{\mathcal{W}}(X)$ by functions in the vector sublattice generated by $\mathcal{M}$ : Let $w \in \mathcal{W}$ be any weight function. Given $\varepsilon>0$, the set

$$
Y=\{y \in X \mid w(y) f(y) \geqslant \varepsilon\}
$$

is closed in $X$ because the function $w f$ is upper semicontinuous, hence $Y$ is compact by the definition of $C_{\mathcal{W}}(X)$. Let $\sigma>0$ be an upper bound for $w$ on $Y$. For every $y \in Y$ there is by Theorem 2.1(b) a function $g_{y} \in \operatorname{span}(\mathcal{M})$ such that

$$
w g_{y} \leqslant w f+\varepsilon \text { and } g_{y}(y)>f(y)-\varepsilon / \sigma .
$$

The latter inequality holds even on an open neighbourhood $U_{y}$ of $y$, and by the compactness of $Y$, finitely many of those neighbourhoods, say $U_{y_{1}}, \ldots, U_{y_{n}}$ cover all of $Y$. Now we choose the function

$$
g=g_{y_{1}} \vee \ldots \vee g_{y_{n}} \vee 0
$$

in the vector sublattice generated by $\mathcal{M} \subset C_{\mathcal{W}}(X)$. Then we have $w g \leqslant w f+\varepsilon$ and $g(y) \geqslant f(y)-\varepsilon / \sigma$ for all $y \in Y$. On multiplying by $w$, the latter yields $w(y) g(y) \geqslant$ $w(y) f(y)-\varepsilon$ for all $y \in Y$. But for $x \in X \backslash Y$ we observe that $w(x) g(x) \geqslant 0 \geqslant$ $\boldsymbol{w}(\boldsymbol{x}) f(x)-\varepsilon$ holds as well. Summarising, this yields

$$
w(x)|f(x)-g(x)| \leqslant \varepsilon \text { for all } x \in X,
$$

hence $p_{w}(f-g) \leqslant \varepsilon$.

The following is a useful tool to identify Korovkin systems in $C_{\mathcal{W}}(X)$ : 
Corollary 2.3. Let $\mathcal{M}$ be a subset of $C_{\mathcal{W}}(X)$. Suppose that for every $x \in X$ such that $w(x)>0$ for at least one weight function $w \in \mathcal{W}$,

(i) there is $g_{x} \in \mathcal{M}$ such that $g_{x}(x) \neq 0$,

(ii) for every $x \neq y \in X$ there is $g_{x, y} \in \operatorname{span}(\mathcal{M})$ such that

$$
g_{x, y} \geqslant 0, \quad g_{x, y}(x)=0 \text { and } g_{x, y}(y)>0 .
$$

Then $\mathcal{M}$ is a Korovkin system for $C_{\mathcal{W}}(X)$.

Proof: It is obvious how the criterion in Corollary 2.2 implies condition (c) of Theorem 2.1 for every function $f \in C_{\mathcal{W}}(X)$ : Let $x \in X$ such that $w(x)>0$ for at least one weight function $w \in \mathcal{W}$ and let $\mu \in M_{B}^{+}(X)$ and $w \in \mathcal{W}$ be such that $w \mu(g)=g(x)$ for all $g \in \mathcal{M}$. For any $x \neq y \in X$ there is a function $g_{x, y} \in \operatorname{span}(\mathcal{M})$ as in (ii); that is, $w \mu\left(g_{x, y}\right)=0$, and the point $y$ cannot be contained in the support of the positive Borel measure $w \mu$. This shows that $w \mu$ is in fact a multiple of the point evaluation in $x$, and the point evaluation itself, as by our assumption (i) there is a function $g_{x} \in \mathcal{M}$ that does not vanish in $x$. Thus we have $w \mu(f)=f(x)$ for all $f \in C_{\mathcal{W}}(X)$, indeed.

In their original work, Bauer and Donner [2] provide several examples for Korovkin systems in $C_{0}(X)$. Many more applications for approximation processes modelled by sequences of positive operators can be found in [1]. We shall conclude this paper with two elementary examples of Korovkin systems for weighted spaces different from $C_{0}(X)$.

EXAMPLE 2.4. (a) Let $X$ be a locally compact Hausdorff space. If $C(X)$ contains a one-to-one function $f$, then for every strictly positive function $g \in C(X)$ the set $\mathcal{M}=\left\{g, g f, g f^{2}\right\}$ fulfils the criterion of Corollary 2.3: Clearly $g \in \mathcal{M}$ satisfies (i), and for a fixed $x \in X$ the function

$$
y \longmapsto g(y)(f(y)-f(x))^{2}
$$

is contained in $\operatorname{span}(\mathcal{M})$, non-negative, vanishes at $x$ but is positive at all $y \neq x$, hence satisfies (ii). Thus, for any choice of a family of weights $\mathcal{W}$ on $X$ such that $\mathcal{M}$ is contained in $C_{W}(X)$, the subset $\mathcal{M}$ is a Korovkin system for $C_{W}(X)$.

(b) Let $X=[0,+\infty)$, and let $\mathcal{W}$ consist of the functions $w_{\alpha}(x)=e^{-\alpha x}$ for all $\alpha>0$. Following part (a), the subset

$$
\mathcal{M}=\left\{f_{k} \mid f_{k}(x)=x^{k} \text { for } k=0,1,2\right\}
$$

is a Korovkin system for $C_{W}(X)$ : Let us illustrate this example with an approximation process modelled by a modified version of the classical Bernstein operators. For a function $f \in C_{W}(X)$ define

$$
T_{n}(f)(x)= \begin{cases}\sum_{k=0}^{n^{2}}\left(\begin{array}{c}
n^{2} \\
k
\end{array}\right) f\left(\frac{k}{n}\right)\left(\frac{x}{n}\right)^{k}\left(1-\frac{x}{n}\right)^{n^{2}-k}, & \text { for } x<n \\
f(n), & \text { for } x \geqslant n .\end{cases}
$$


These operators $T_{n}$ are clearly linear and positive on $C_{\mathcal{W}}(X)$. With some straight forward computations one may check the following:

$$
\begin{array}{ll}
T_{n}\left(f_{0}\right)(x)=1 & \text { for all } x \in[0,+\infty), \\
T_{n}\left(f_{1}\right)(x)=x & \text { for all } x<n, \\
T_{n}\left(f_{2}\right)(x)=\frac{n^{2}-1}{n^{2}} x^{2}+\frac{1}{n} x & \text { for all } x<n .
\end{array}
$$

This shows in particular that $T_{n}\left(f_{k}\right) \rightarrow f_{k}$ for $k=0,1,2$ in the topology of $C_{\mathcal{W}}(X)$. Furthermore, one may check that the sequence $\left(T_{n}\right)_{n \in \mathbb{N}}$ is indeed equicontinuous, as for any $f \in C_{\mathcal{W}}(X)$ and $\alpha>0$

$$
|f| \leqslant e^{\alpha x} \text { implies that }\left|T_{n}(f)\right| \leqslant e^{(e \alpha) x} ;
$$

that is

$$
p_{w_{\alpha}}\left(T_{n}(f)\right) \leqslant p_{w_{(\alpha / e)}}(f)
$$

for all $f \in C_{\mathcal{W}}(X)$ and $n \in \mathbb{N}$. Thus Theorem 2.1 applies, and we may conclude that $T_{n}(f) \rightarrow f$ for all $f \in C_{\mathcal{W}}(X)$.

\section{REFERENCES}

[1] F. Altomare and M. Campiti, Korovkin type approximation theory and its applications, de Gruyter Studies in Mathematics 17 (Walter de Gruyter, Berlin, New York, 1994).

[2] H. Bauer and K. Donner, 'Korovkin approximation in $C_{0}(X)$ ', Math. Ann. 236 (1978), 225-237.

[3] A.D. Gadzhiev, 'Theorems of Korovkin type', Math. Notes 20 (1976), 995-998.

[4] A.D. Gadzhiev, 'Positive linear operators in weighted spaces of functions in several variables', (in Russian ), Izv. Akad. Nauk. Azerbaídzan SSSR ser. Fiz.-Tekhn. Mat. Nauk 4 (1980), 32-37.

[5] L. Nachbin, Elements of approximation theory (D. Van Nostrand Co., Princeton, NJ, 1967).

[6] J.B. Prolla, Approximation of vector-valued functions, Mathematical Studies 25 (North Holland, 1977).

Department of Mathematics

University of Bahrain

PO Box 32038

Bahrain

e-mail: roth@sci.uob.bh 